\title{
Du rouge au bleu Marine : évolution des communes rurales à l'ouest du département de l'Hérault
}

From red to Marine blue: an evolution of rural administrative subdivisions West of the Hérault section

\section{Marie-Paule Crochet-Théry}

\section{(2) OpenEdition}

Journals

Édition électronique

URL : http://journals.openedition.org/add/1470

DOI : $10.4000 /$ add. 1470

ISSN : 2606-1988

Éditeur

Presses universitaires de Rouen et du Havre

Édition imprimée

Date de publication : 1 mai 2019

Pagination : $57-87$

ISBN : 979-10-240-1293-3

ISSN : 1955-0855

\section{Référence électronique}

Marie-Paule Crochet-Théry, « Du rouge au bleu Marine : évolution des communes rurales à l'ouest du département de l'Hérault », Les Annales de droit [En ligne], 13 | 2019, mis en ligne le 09 décembre 2019, consulté le 26 janvier 2021. URL : http://journals.openedition.org/add/1470 ; DOI : https://doi.org/ ERREUR PDO dans /localdata/www-bin/Core/Core/Db/Db.class.php L.34 : SQLSTATE[HY000] [2006] MySQL server has gone away

Ce document a été généré automatiquement le 26 janvier 2021.

Presses universitaires de Rouen et du Havre 


\section{Du rouge au bleu Marine : évolution des communes rurales à l'ouest du département de l'Hérault}

From red to Marine blue: an evolution of rural administrative subdivisions West of the Hérault section

Marie-Paule Crochet-Théry

\section{NOTE DE L'AUTEUR}

Les premiers mots du titre font référence à un article d'Emmanuel Négrier paru en 2014 : « Une vague bleue en Midi rouge. Les élections 2014 en LanguedocRoussillon ", Pôle Sud, vol. 41, nº 2, 2014, p. 211, https://www.cairn.info.

1 L'étude porte sur 46 petites communes rurales ${ }^{2}$ précédemment analysées électoralement lors d'un travail de thèse soutenu en mars $2017^{3}$. Examiner la montée en puissance des votes en faveur du Front national (FN) dans cet espace particulier présentait en début de recherche l'avantage de travailler sur un champ relativement peu exploité en 2011, même si Bernard Schwengler avait publié en 2003 un article sur un vote rural d'extrême droite dans les très petites communes rurales de la Meuse et des Vosges ${ }^{4}$ et si Emmanuel Pierru et Sébastien Vignon avaient, en 2007 et 2008, étudié les conséquences politiques des mutations des modes de vie du monde rural dans le département de la Somme, à partir également de très petits villages 5 .

2 Toutes les localités étudiées présentent des caractéristiques communes: aucune ne dépassait les 500 électeurs inscrits en 2012 ; toutes se trouvent économiquement dans l'orbite de Béziers, hors de l'attraction montpelliéraine, dans une zone restée profondément rurale hormis, dans l'ex-canton de Bédarieux, quelques communes caractérisées par un passé récent minier ou industriel. 
3 Toutes, depuis les élections législatives de 2012, se retrouvent administrativement dans la cinquième circonscription de l'Hérault où, à l'issue du premier tour de ce scrutin et à la surprise générale - y compris celle des responsables héraultais du $\mathrm{FN}$ - une très jeune candidate Rassemblement bleu Marine $(\mathrm{RBM})^{6}$ se retrouva seule en lice face à un socialiste, député sortant, conseiller général, maire de Saint-Pons-de-Thomières, depuis longtemps solidement installé dans son fief. La question de la réalité d'une présence du FN, dans ces terres jugées jusque-là assez hostiles aux idées frontistes par la fédération FN 34, était alors posée.

4 Toutes appartiennent historiquement au "Midi rouge ${ }^{7}$ ». Lors des élections présidentielles de 1981, qui ont servi d'année de référence pour le travail de thèse, les trois quarts de ces villages avaient voté majoritairement socialiste ou communiste le 26 avril's. Le Parti communiste y a conservé d'ailleurs des noyaux d'électeurs fidèles jusqu'à la fin du $\mathrm{xx}^{\mathrm{e}}$ siècle 9 .

5 Sur ces communes, depuis des décennies acquises aux valeurs républicaines de la Gauche, a longtemps pesé l'hypothèse de l'impossibilité d'un vote d'extrême droite, d'un vote Le Pen. De fait, jusqu'en 2010, cette hypothèse de résistance au vote FN ne pouvait être écartée. L'ensemble des communes présentait à chaque élection des scores en faveur du FN toujours inférieurs à ceux du département et très en retrait vis-à-vis de ceux obtenus en zone littorale, même lors de l'élection présidentielle du 21 avril $2002^{10}$. Fait remarquable, Jean Saint-Josse, de Chasse, pêche, nature et tradition (CPNT), était alors seulement à un point du candidat frontiste. 2007 fut une année noire pour le parti, tant aux élections présidentielles que législatives ${ }^{11}$. Lors des élections européennes de 2009, le FN ne représentait guère plus que $3 \%$ des inscrits. En 2010, lors des élections régionales, si particulières en Languedoc-Roussillon ${ }^{12}$, la candidate FN, France Jamet ${ }^{13}$, présente au second tour, totalisait moins de $10 \%$ des voix ${ }^{14}$.

6 Enfin, ces localités étaient, et sont toujours, des lieux où les questions de la présence ou la proximité immédiate d'une population immigrée, ainsi que des problèmes d'insécurité, ne se posaient pas ou peu, comme le reconnaissait elle-même la candidate mariniste aux législatives de $2012^{15}$. Ce n'étaient pas davantage des terres où les Piedsnoirs, rapatriés d'Algérie après 1960, avaient choisi de s'installer en nombre. Ces facteurs ne pouvaient donc être comptés parmi les causes susceptibles d'y favoriser une implantation frontiste.

$7 \quad$ Une étude systématique des résultats obtenus par l'ensemble des partis en présence sur la totalité des consultations électorales depuis $1984^{16}$ a pu, dans un premier temps, permettre de mesurer objectivement l'évolution des votes FN et de la resituer, à la fois dans le contexte de l'offre partisane et dans celui de l'époque, grâce à une étude systématique de la presse locale ${ }^{17}$.

8 La montée en puissance du parti frontiste, observée nettement après 2011, obligea à rechercher dans quelle mesure des facteurs sociogéographiques et économiques avaient pu contribuer à la profonde transformation d'un espace électoral dominé encore au début des années quatre-vingt par le Parti communiste et par le Parti socialiste. 


\section{L'évolution électorale des villages et la place du FN depuis 1984}

9 On a cherché à établir dans un premier temps la réalité de l'implantation du FN, ou plus exactement du vote $\mathrm{FN}$, dans les communes choisies en privilégiant l'allure générale de la progression et la place prise par le FN au fil du temps.

\subsection{L'allure générale de la progression}

10 Celle-ci ne s'est pas faite linéairement. Elle a varié selon la nature des consultations. Parmi tous les scrutins, les élections présidentielles occupent une place à part, caractérisée par des scores toujours plus élevés, tant en suffrages exprimés qu'en nombre d'inscrits.

11 D'importance quantitative faible quant au nombre de villages concernés en 1984, date de l'apparition des premiers votes FN, la position du parti de Jean-Marie Le Pen progresse modérément, hormis lors de l'élection présidentielle de 1988, jusque 1989, puis stagne jusque 1997, y compris lors de l'élection présidentielle de 1995. De 1998 à 2009 on assiste à une évolution en dents de scie, où alternent des scrutins à forte progression - telle l'élection présidentielle de 2002 avec un score à près de $20 \%$ le 5 mai, ou bien les régionales de 2004 qui voient le $\mathrm{FN}$ en position d'imposer une triangulaire - avec des scrutins marqués par d'importantes régressions, qu'il s'agisse des européennes de 1999 ou des législatives de 2007.

12 La situation change à partir de 2010, comme l'indiquent les courbes des résultats obtenus par le FN. Le gain en voix de la candidate frontiste au second tour des élections régionales est un phénomène nouveau. À partir de 2012, on assiste à une progression continue, marquée par des scores toujours supérieurs à $20 \%$, quels que soient le type d'élection ou le mode de scrutin. Ce phénomène n'est d'ailleurs pas propre à l'Ouest héraultais, il se manifeste dans l'ensemble de la région Languedoc-Roussillon. Selon Emmanuel Négrier :

Lors des élections régionales de 2010 , le FN passe respectivement de $8,2 \%$ des voix à $18,7 \%$ dans les communes de moins de 200 habitants et de $10,4 \%$ à $21,7 \%$ dans les communes comptant entre 200 et 500 inscrits. Cela représente une progression en pourcentages de plus de $100 \%$, qui plus est dans les zones où la participation est la plus importante à ce scrutin, comme à celui des élections régionales d'ailleurs ${ }^{18}$.

Cette progression marque cependant le pas lors des législatives de 2017, où les gains de pourcentage de voix sont non seulement à mettre en relation avec une envolée inédite des votes blancs et des abstentions, mais aussi avec le constat d'une baisse de près de quatre points par rapport au second tour des législatives précédentes si l'on raisonne en nombre d'inscrits. 
Courbes comparées des évolutions électorales de 1984 à 2017. Remarque : Le pourcentage des abstentions indiqué pour les élections législatives de 2017 inclut les votes nuls et les votes blancs. Seul il atteint 50,63 \%. Courbes réalisées à partir de données quantitatives officielles.
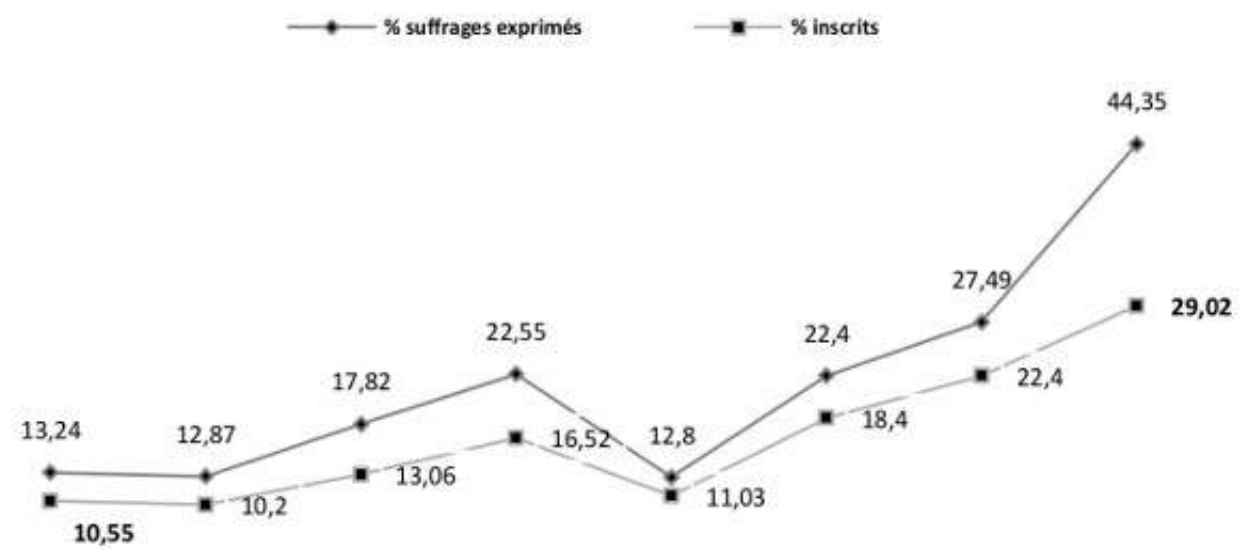

\begin{tabular}{lllllllllll}
\hline 1988 & 1995 & $21 / 04 / 02$ & $05 / 05 / 2002$ & 2007 & 2012 & $24 / 04 / 2017$ & $07 / 05 / 2017$
\end{tabular}

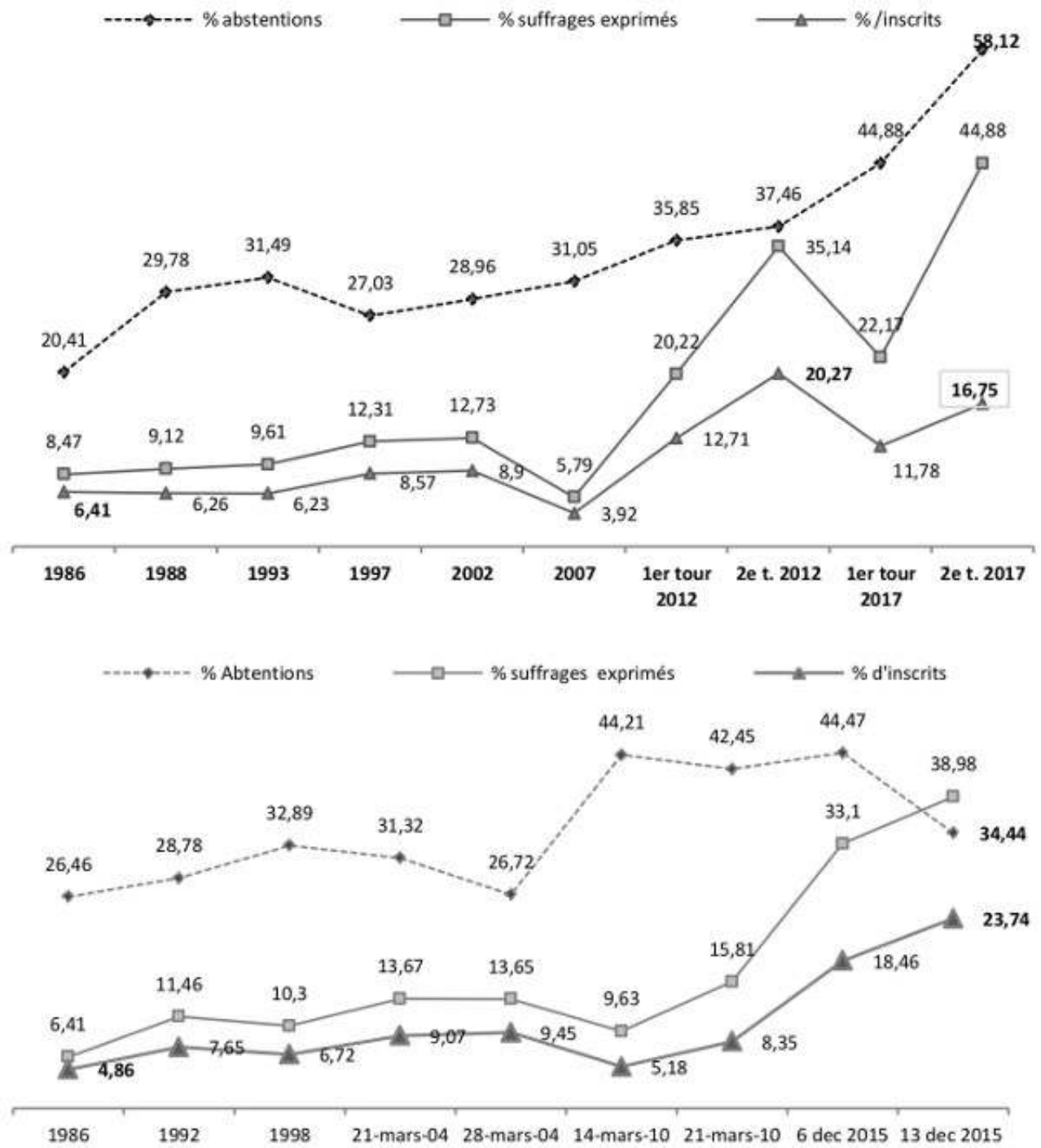



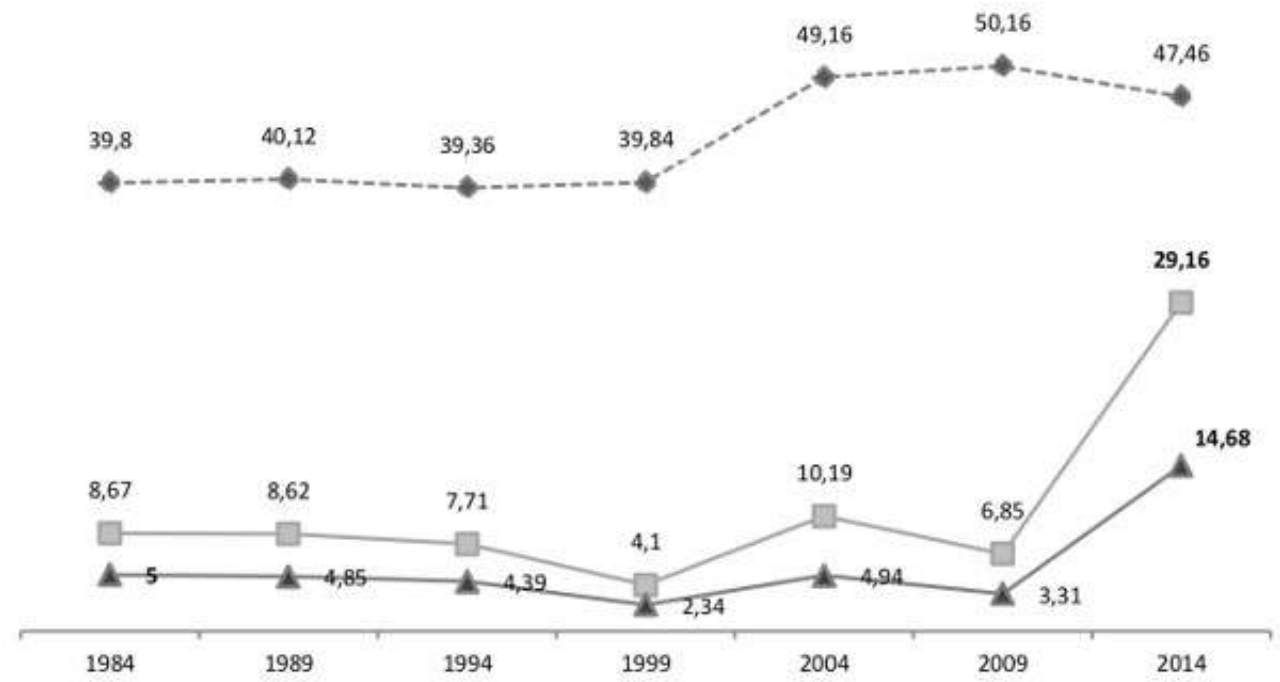

\subsection{La progression spatiale et la notion de "noyau dur »}

d'ensemble, la notion d'une progression spatiale, s'est imposée la nécessité de trouver un critère objectif qui peut permettre de mesurer cette avancée. Le terme de «noyau dur " définit le nombre de communes constamment à plus de $15 \%$ pour un type d'élection donnée. L'étude de sa croissance, à la fois quantitative ${ }^{20}$ et qualitative ${ }^{21}$ en fonction des différents types de scrutins ${ }^{22}$, a permis de voir quand celle-ci atteint « une masse critique " telle qu'il devient possible objectivement de parler d'implantation électorale du FN; surtout quand cette extension est observée sur différents types de consultations.

\subsubsection{Le cas particulier des élections présidentielles}

${ }^{23}$ L'étude des résultats des scrutins au premier tour présidentiel permet de constater l'existence précoce d'un noyau dur. Dès 1988, 20 villages (sur 46) donnent au FN des scores au-dessus de $15 \%$. Certains ont même des taux élevés ${ }^{24}$. Au fil des élections, d'autres localités vont venir grossir ce premier noyau. Leur nombre, jamais inférieur à 17 , s'élève à 30 en 2002, à 28 en 2012, à 37 le 23 avril 2017 et à 43 le 7 mai. À ce stade, c'est presque l'ensemble des communes qui est concerné. Seuls trois villages affichent un score frontiste inférieur à $15 \%$.

L'accroissement constaté est aussi qualitatif. Si l'on excepte l'élection présidentielle de 2007, le poids électoral des communes du noyau dur s'affermit au fur et à mesure des consultations. Les votes de ces villages en faveur de Jean-Marie Le Pen, qui jusque 1995 représentaient environ $7 \%$ du total des suffrages exprimés par rapport à l'ensemble des communes, doublent en 2001, pour atteindre chez sa fille près de $30 \%$ le 23 avril 2012 et plus de $44 \%$ le 7 mai $2017^{25}$. 

d'appréhension de leurs intérêts personnels au plan local Cette stratégie électorale explique en grande partie le manque de renouvellement des conseillers généraux lors des élections cantonales et les mauvais scores récurrents obtenus par les candidats FN pour ce type de scrutin. L'emprise économique du conseil général et donc du conseiller général ${ }^{38}$ sur les villages ${ }^{39}$ est telle que, si l'on examine depuis 1985 l'évolution des votes des communes composant le noyau dur pour d'autres élections, on ne constate une progression des votes frontistes que dans une petite minorité d'entre elles. Dans les autres, les scores obtenus demeurent modestes, voire faibles. 

prend comme exemple le canton de Saint-Pons-de-Thomières qui compte désormais les cantons de Capestang, d'Olargues, d'Olonzac, de La Salvetat-sur-Agout et de SaintChinian, il renferme plus de la moitié des villages étudiés. Le binôme mariniste ${ }^{40} \mathrm{est}$, au premier tour, à 1 point seulement ${ }^{41}$ de celui créé par le conseiller sortant, alors député et ancien maire de Saint-Pons, le socialiste Kléber Mesquida qui l'emportera finalement. Le 29 mars, le tandem FN recueille $40,76 \%$ des voix, soit près d'un quart des inscrits. Trois villages lui ont donné la majorité. président de région, huit jours après le second tour du scrutin ${ }^{42}$. Les listes menées par les présidents sortants, de Jacques Blanc (en 1992, 1998) à Georges Frêche (en 2010), ont, de fait, toujours obtenu des résultats qui allaient au-delà de l'influence de leur parti réciproque.

Si l'on se réfère aux résultats obtenus par le FN lors des six consultations ${ }^{43}$ ayant eu lieu entre 1986 et 2015, on constate une première progression spatiale en 1992. Elle coïncide avec le premier renouvellement de la présidence de Jacques Blanc qui, élu en 1986 en partie grâce aux voix des conseillers frontistes ${ }^{44}$, avait établi un climat de bonnes relations avec eux durant son premier mandat ${ }^{45}$. L'importance numérique et spatiale du FN reste toutefois limitée jusqu'aux élections de décembre 2015 où la liste de Louis Aliot remporte $33,1 \%$ des suffrages au premier tour ${ }^{46}$ et $38,99 \%$ le 13 décembre. Dans sept communes, les votes frontistes ont dépassé la majorité des suffrages exprimés. La baisse visible des abstentions lors du second tour dans les communes où le FN avait réalisé ses meilleurs scores le 6 décembre montre un effet de mobilisation des électeurs marinistes.

Les élections européennes sont le troisième scrutin de type intermédiaire. Pour des raisons différentes de celle des cantonales ${ }^{47}$, elles présentent avec celles-ci des analogies frappantes. L'échec récurrent des listes FN jusque $2014^{48}$ fait que seuls trois petits villages ${ }^{49}$ votent depuis 1984 presque toujours à plus de $15 \%$ pour les listes frontistes.

Le contraste est saisissant avec les élections du 25 mai 2014 où la liste RBM de Louis Aliot remporte près de $30 \%$ des suffrages, dépassant de quatre points les scores obtenus dans la circonscription Sud-Ouest. Plus de $78 \%$ des communes ont voté FN à plus de $15 \%$. Montels a choisi la liste frontiste à $54,46 \% .7$ villages ont des scores qui dépassent $40 \%{ }^{50} .13$ sont à plus de $30 \%$. Les bons résultats réalisés en 2012 par le $\mathrm{FN}$, tant aux élections présidentielles que législatives, et le battage médiatique à propos des municipales de 2014 ont réussi à créer une dynamique favorable.

\section{3. Évolution de la place du FN dans le paysage politique des villages}

Présent depuis 1984, le parti de Jean-Marie Le Pen a construit son influence dans les localités étudiées, en prenant des voix d'abord à la droite puis à la gauche. Dès la fin des années 1980, il participe à la déstabilisation du paysage politique ${ }^{51}$ pour en devenir un acteur majeur ces sept dernières années. Pourtant, l'étude des variations des scores communaux et des fluctuations du noyau dur, effectuée sur plus de trente ans, montre que la grande masse des électeurs du FN est éminemment fluctuante malgré l'importante progression des votes constatée à chaque scrutin après 2011. Le 
phénomène est observable au sein même des communes répertoriées comme les plus favorables, au-delà même des résultats catastrophiques des élections de 2007 ou de ceux déjà évoqués des cantonales et des européennes ${ }^{52}$. Dans les villages étudiés comme ailleurs, pour reprendre l'expression de Patrick Lehingue, "l'électorat frontiste n'existe $\operatorname{pas}^{53} »$. Toutefois, et sans que cela modifie radicalement ce constat, il est possible que les scores élevés obtenus le 22 avril 2012 et lors des législatives qui ont suivi les présidentielles aient eu un effet d'entrainement et que l'on assiste pour les scrutins suivants à une plus grande régularité de votes partisans, dans la mesure où l'on constate une diminution du nombre des abstentions ${ }^{54}$ en 2014 et $2015^{55}$ dans les communes où les listes marinistes sont à plus de $30 \%$.

\section{2. Étude de cas particuliers}

28 Une étude ciblée sur quelques villages offre un double avantage. Elle permet un suivi plus fin de l'évolution des votes. Dans l'objectif d'une recherche explicative, elle offre aussi l'occasion de pouvoir confronter l'interprétation de la montée des votes FN par des causes classiquement invoquées pour l'expliquer, avec une réalité de terrain, fut-il limité, dans la mesure où ces communes sont bien représentatives de l'ensemble des sites choisis.

Le travail a porté sur 5 villages et deux types de scrutins : les élections présidentielles et régionales. 4 sont des communes rurales ${ }^{56}$, la $5^{\mathrm{e}}$, Graissessac, est un village minier dont la situation démographique et économique a changé radicalement avec la fermeture des puits dès 1964 et l'arrêt de toute exploitation à ciel ouvert en 1994. Toutes ces localités s'inscrivent dans une longue tradition républicaine de gauche, comme la majorité des communes de l'Ouest héraultais étudiées. Dans deux d'entre elles, Margon et Pierrerue, il a été possible de mener des entretiens.

\subsection{L'évolution des votes FN}

Entre $1986^{57}$ et 2017, l'examen des résultats montre une évolution différente dans la progression des votes $\mathrm{FN}$ dans les 5 villages.

31 Quasi inexistant dans tous en 1984, le FN devient en 2012 la seconde force politique à Margon et à Pierrerue et la première en 2017, mais de façon plus affirmée à Margon ${ }^{58}$. Il est, à cette date, la seconde force politique à Graissessac, 7 points derrière le Parti de gauche de Jean-Luc Mélenchon. Il est à la troisième place à Montouliers où, jusqu'en 2012, il peine à émerger du lot des "petits partis ${ }^{59}$ ». Il reste en 2017 à Pardailhan une force politique marginale.

\subsection{1. À Graissessac}


Évolution des votes FN aux élections présidentielles dans les cinq villages de 1988 à 2012. On peut noter que les résultats de la présidentielle du premier tour de 2017 se placent sur la trajectoire des courbes de tendance avec même une accentuation des scores.

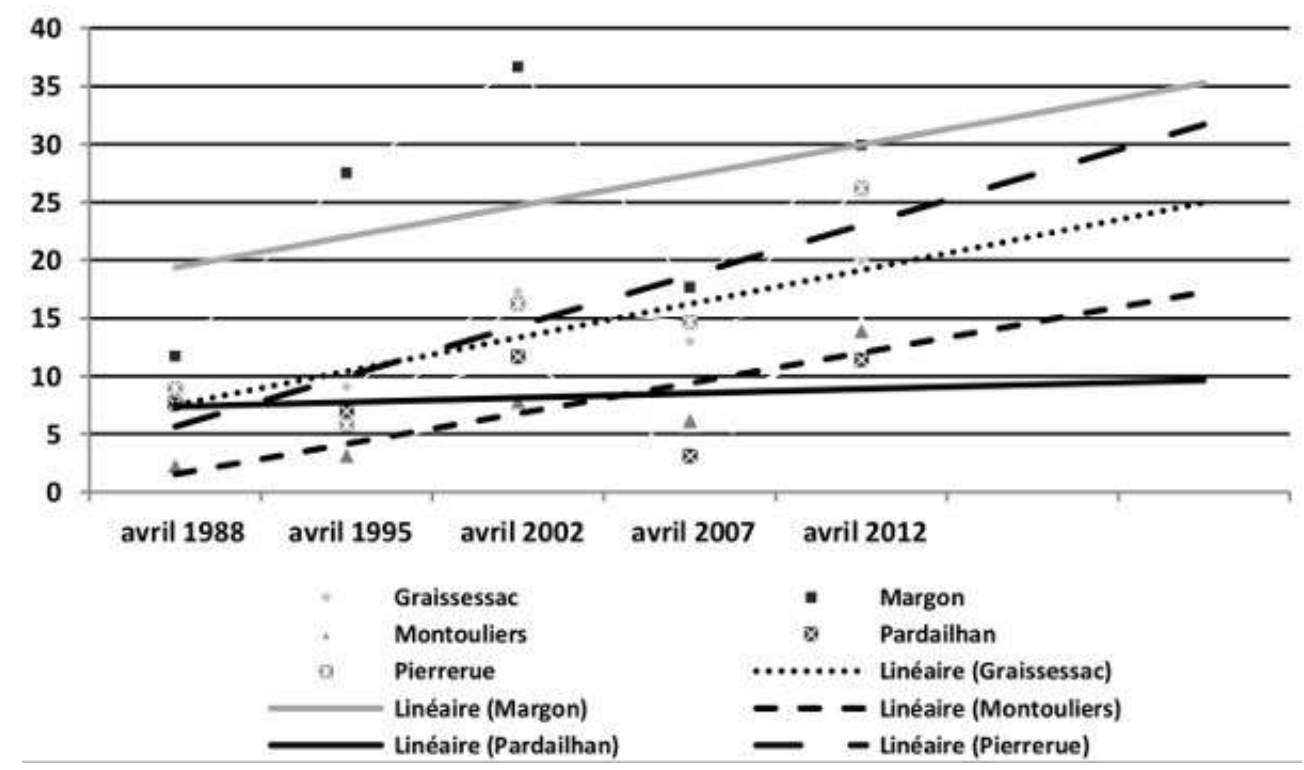

Dans cet ancien village minier à la reconversion difficile ${ }^{60}$, qui a vu sa population s'effondrer jusqu'au milieu des années 1980, le FN a peu de prise jusqu'à l'élection présidentielle de 2002 où Jean-Marie Le Pen recueille un peu plus de $17 \%$ des voix. Le PC qui représentait en 1988 pratiquement $30 \%$ des voix, chute à $11 \%$. Après 2002 les scores frontistes vont rester à deux chiffres à chaque présidentielle ${ }^{61}$, en hausse nette depuis 2012. Les régionales de 2004 marquent la même tendance à la hausse des voix pour le FN qui recueille 14,53\% des suffrages, une hausse enrayée en 2010 en faveur de Georges Frêche ${ }^{62}$. Jusqu'à 2014 néanmoins le FN reste toujours en deçà des scores atteints par le PS.

\subsection{2. À Margon}

Le village se caractérise par un accroissement particulièrement rapide de sa population durant la première décennie du $\mathrm{Xxl}^{\mathrm{e}}$ siècle $^{63}$. Lors du recensement de 2011 plus de la moitié des Margonais n'habitait pas la commune quatre ans auparavant. S'ensuit un large renouvellement du corps électoral. Cette commune viticole de plaine, au sud-est de la zone étudiée, est parmi les cinq villages celle où les électeurs donnent le plus de voix au parti frontiste. La courbe des pourcentages s'élève, en quatre élections présidentielles, d'un peu plus de $11 \%$ en 1988 à près de $30 \%$ en $2012^{64}$, à $38,24 \%$ le 23 avril 2017 (avec une abstention à 15,46\%) et à 53,93\% le 7 mai (abstentions 17,27\%, bulletins blancs $9,71 \%$ ). Elle monte, en quatre élections régionales, de moins de $4 \%$ à pratiquement $23 \%$. La baisse constatée en 2010 est éphémère et en 2015 les scores en faveur de la liste RBM de Louis Aliot vont dépasser $35 \%$. Il devient possible de parler pour Margon d'une véritable implantation frontiste.

\subsection{3. À Montouliers}

34 Village vinicole au sud-ouest de la zone étudiée, en limite du département de l'Aude, Montouliers possède un secteur agricole important puisqu'en 2013, il génère encore $43,3 \%$ des emplois salariés existants sur place. Il fait partie des communes où le FN 
peine à trouver des voix. Crédité de moins de $2 \%$ en 1988, il progresse difficilement à chaque élection présidentielle jusque 2012 et ne dépasse les $20 \%$ qu'en $2017^{65}$, pour atteindre $29,06 \%$ le $7 \mathrm{mai}^{66}$. Son meilleur score obtenu lors des élections régionales est de $5 \%$ en 2010. Dans ce village, le parti socialiste reste dominant ${ }^{67}$ jusqu'à ce que l'élection présidentielle de $2017^{68}$ bouleverse la donne. Lors des présidentielles précédentes les scores des candidats socialistes sont toujours restés supérieurs ou sensiblement égaux à $30 \%{ }^{69}$. Ceux atteints lors des élections régionales sont encore plus impressionnants puisqu'ils se situent 4 fois sur 5 entre $50 \%$ et $60 \%$.

\subsection{4. À Pardailhan}

35 Ce village, qui porte un nom homonymique des montagnes qui l'entourent, se situe dans la zone montagneuse du département. On y pratique la polyculture et un peu d'élevage sur une terre assez pauvre. Le secteur agricole, bien qu'en baisse, représente en $201329,17 \%$ des emplois sur place ${ }^{70}$.

Lors des élections présidentielles, le FN se situe encore aujourd'hui dans la zone des partis à faible audience. Le 21 avril 2002, avec une abstention légèrement inférieure à $24 \%$, Jean-Marie Le Pen n'avait réuni que $11,65 \%$ des voix ${ }^{71}$. En 2012, Marine Le Pen en obtient $11,38 \%$. En 2017, son score lors du premier tour est à peine plus élevé : $12,73 \%^{72}$. En regard, les candidats socialistes sont constamment au-dessus de $30 \%$ des suffrages jusqu'à l'élection de $2017^{73}$.

37 L'étude des élections régionales confirme l'échec jusqu'en 2010 des candidats frontistes, constamment en dessous de $5 \%$ des voix, hormis en 1998 où Jean-Claude Martinez obtient $6,58 \%$ des suffrages. Mais pour ces scrutins, contrairement à Montouliers, Pardailhan ne peut être compté comme un bastion socialiste. En effet, si le PS y domine le paysage politique en 1986 et à partir de 2004 avec l'arrivée de Georges Frêche à la tête de la région, durant la présidence régionale de Jacques Blanc, lors des élections régionales de 1992 et de 1998, c'est la droite UDF-RPR qui remportait le plus de suffrages ${ }^{74}$.

\subsection{5. À Pierrerue}

Village viticole près de Saint-Chinian, Pierrerue perd des habitants jusqu'en 2006. En 2012, le niveau démographique de 2012 est à peine supérieur à celui de $1982^{75}$. C'est un village à la population vieillissante, où en 2011 près de $68 \%$ des habitants avaient plus de $45 \mathrm{ans}^{76}$. Il fait partie avec Graissessac des communes où l'on observe une grande stabilité de résidence. Le secteur agricole y est encore particulièrement important puisqu'il fournit $46,44 \%$ des emplois sur place. Humainement, selon l'une des personnes interviewées, c'est un village où tout le monde se connaît et où l'on se méfie de l'étranger. La gestion du village reste exclusivement l'affaire des natifs. Le FN y poursuit une progression régulière lors des élections présidentielles. Parti de 8,93\% en 1988, il atteint $26,18 \%$ en 2012 avec une perte de moins de 2 points en 2007 par rapport aux scores de 2002. Il est le 23 avril 2017 sensiblement au même pourcentage (26,94\%) qu'en 2012, mais le 7 mai c'est à $47,02 \%$ que les Pierrerunais choisissent Marine Le Pen, le total des abstentions et des votes blancs étant inférieur à $27 \%$. La lecture des scores des candidats $\mathrm{FN}$ lors des élections régionales montre un taux de votes frontistes qui progresse à partir de $2004^{77}$ pour dépasser les $20 \%$ au second tour de l'élection de 2010. En 2015, la liste Louis Aliot obtient 31,91\% des suffrages au 
premier tour et $40,91 \%$ le 13 décembre, avec une abstention légèrement inférieure à $30 \%(28,82 \%)$. Quant au paysage politique, si, comme à Montouliers ou même Pardailhan, le CPNT y fait de bons scores dans la décennie $1990^{78}$, on constate à la même période que la dominance des partis de gauche, PC et PS, s'amenuise au fil des élections, avant de remonter le temps de la présidence de région de Georges Frêche ${ }^{79}$.

\subsection{Recherche d'explications}

À moins de se contenter de croire que l'arrivée de Marine Le Pen à la tête du parti et le succès de la dédiabolisation, largement relayé par la presse écrite et télévisée, et les instituts de sondage ${ }^{80}$ ont convaincu les habitants des villages au point de donner au FN des scores toujours supérieurs à $20 \%$, il convient de rechercher plutôt les ressorts de cet accroissement brusque des votes frontistes depuis 2011 dans des causes en rapport direct avec l'environnement économique et la vie quotidienne de ces électeurs.

\subsubsection{L'explication par les difficultés socio-économiques}

Parmi les facteurs classiquement évoqués viennent en premier les difficultés socioéconomiques.

En 2013, Pascal Perrineau, parlant de la France "périphérique ${ }^{81}$ " dans le chapitre consacré à la fracture territoriale, constate que « la forte pénétration du FN [...] renvoie à un certain nombre de difficultés caractéristiques de ces territoires [...] un niveau de revenu plutôt faible, une fragilité du tissu économique local [...] la disparition fréquente de certains services publics [...] un choix d'habitation qui peut être "contraint" [dans] ces territoires constitués de petites communes, où l'on compte peu d'immigrés ». Il fait remarquer combien "le Front national avait su développer un vocabulaire et des référents idéologiques qui parlent aux "perdants" de la mondialisation ${ }^{82}$ ». Déjà en 2010, Christophe Guilluy évoquait « ces zones rurales où se conjuguent bas salaires, chômage et grande précarité83 $"$.

Il est aisé est de constater que l'ouest héraultais rural entre parfaitement dans une telle description. La précarité y touche inégalement les communes rurales, mais tend à augmenter. En 2012, plus de la moitié des communes étudiées se trouvait classée en zone de revitalisation rurale. Dans le département de l'Hérault, en 2013, l'indice de pauvreté atteint $19,8 \%^{84}$. En dépit des mécanismes de redistribution, un Héraultais sur cinq vit en dessous du seuil de pauvreté.

On doit néanmoins constater que ces phénomènes sont antérieurs à 2010 et ne peuvent donc expliquer à eux seuls la montée en puissance du FN constatée après cette date.

Déjà au moment des campagnes électorales, en 1994-1995, la lecture de documents de l'Insee et d'articles de presse montrait l'aspect structurel de ces difficultés, un aspect que la crise économique de 2008 n'a pu qu'aggraver. En 2009, une note d'analyse sur "la pauvreté dans le monde rural ${ }^{85}$ ", élaborée au moment de la mise en place du RSA dans le cadre départemental, ciblait tout un catalogue de facteurs socio-économiques de mise en place de la précarité, telle la diminution, voire la disparition de services publics et des commerces locaux, l'absence ou la faiblesse des transports en commun, le coût des déplacements pendulaires, l'isolement social et le vieillissement d'une partie importante de la population. Il y était dit que, souvent peu visible par peur d'une stigmatisation par le voisinage, la pauvreté, plus fréquente dans les communes 
isolées ${ }^{86}$, atteignait particulièrement les personnes âgées, les familles monoparentales, les jeunes professionnellement peu qualifiés et les néoruraux, venus pour trouver un logement à prix abordable ${ }^{87}$.

Il se pourrait cependant qu'une aggravation de la situation, alliée à un ciblage de l'électorat rural dans le discours mariniste, puisse expliquer l'augmentation constatée des votes frontistes. L'étude, dans le cadre des cinq villages précédemment décrits, de deux marqueurs, le chômage et la précarité, appréhendée par le biais des données fiscales, permet d'éclairer la situation.

\subsubsection{Le rôle de la montée du chômage}

Faiblesse des salaires et importance des taux de chômage expliquent aussi l'importance particulièrement élevée de la précarité en zone rurale. Différents documents de l'Insee montrent à la fois la grande dépendance économique vis-à-vis de Béziers, bassin d'emploi de 70 \% des villages, et la fragilité économique de ce bassin et de l'ensemble de la zone où, structurellement, une insuffisance de postes de cadres se conjugue avec une majorité d'emplois tertiaires durement touchés par la crise après 2008. Le chômage y est majoritairement plus élevé que dans le département ${ }^{88}$, lui-même à plus de 5 points au-dessus de la moyenne nationale. Les cinq villages étudiés ne dérogent pas, avec des nuances, à ce constat.

Un travail sur les données de l'Insee, prises sur 15 ans et relatives aux cinq villages ciblés, montre l'étendue du problème du chômage et son aggravation par la crise à partir de 2008. En 2014, dans l'ancien village minier de Graissessac, le taux de chômage atteint $25 \%$. Pierrerue fait figure d'exception avec un chômage stable à 10,3 \% .

\subsubsection{Le rôle de la précarité}

Évolution du taux de chômage 1999-2014. Travail effectué à partir des données de l'Insee, chiffres clés. (Pour 2014, chiffres des données actualisées le 12 octobre 2017). Les taux pour le Languedoc-Roussillon sont ceux du second trimestre 2013.

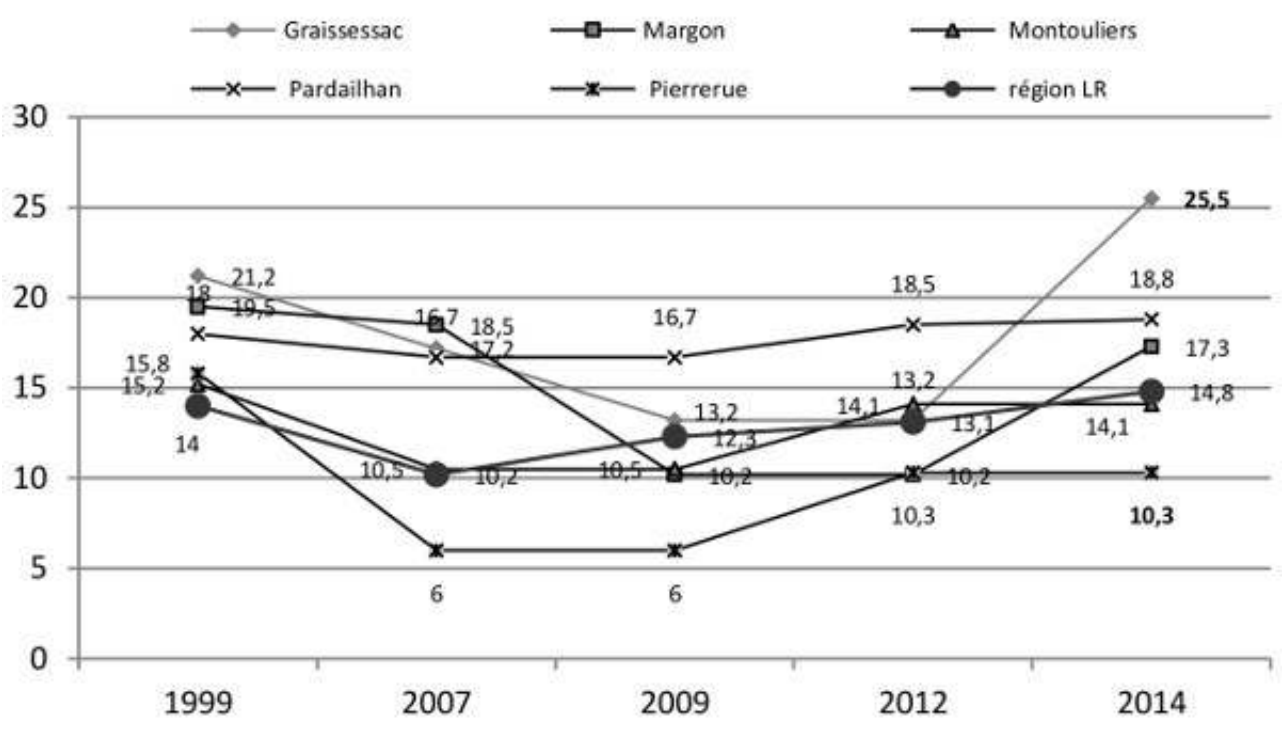


Les données fiscales permettent indirectement de cerner l'ampleur du phénomène pour une localité. La première donnée à considérer est le rapport entre les foyers fiscaux imposables et non imposables ${ }^{89}$.

\begin{tabular}{|l|l|l|l|l|l|}
\hline \multicolumn{6}{|l|}{ Pourcentage des ménages assujettis à l'impôt de 2006 à 2012 à Graissessac, Margon et Pardailhan } \\
\hline Communes & 2006 & 2007 & 2008 & 2009 & 2012 \\
\hline Graissessac & 35,1 & 35,8 & 35,8 & 36,5 & 45,4 \\
\hline Margon & 44,7 & 41,2 & 43,8 & 44,7 & 52,4 \\
\hline Pardailhan & 28,8 & 28,3 & 32 & 35 & 34,6 \\
\hline $\begin{array}{l}\text { Travail personnel à partir des données statistiques fournies par l'Insee. Années 2006-2009: source } \\
\text { DGFIP, impôt sur le revenu des personnes physiques (Insee, chiffres clés). Pour l'année 2012, source } \\
\text { Caisse d'épargne : Diagnostic socio-économique et financier, le bilan santé des communes 2015. }\end{array}$ \\
\hline
\end{tabular}

En 2012, les communes étudiées ont un ratio inférieur au ratio national $(56,4)$ et à celui de la région Languedoc-Roussillon $(49,5)$. Seul, le village de Margon surpasse légèrement le ratio du département $(51,2)^{90}$. À Graissessac, à Montouliers et à Pierrerue, entre 4 et 5 foyers sur 10 sont concernés ${ }^{91}$. À Pardailhan, à peine plus d'un tiers des foyers fiscaux est imposé (34,6\%), malgré une légère augmentation du pourcentage du nombre des foyers assujettis à l'impôt depuis 2006.

Une étude comparée sur trois villages montre d'ailleurs que cette tendance n'est pas propre à cette commune.

51 Le second facteur à prendre en compte est le revenu net moyen par foyer fiscal imposable et non imposable.

52 En 2009, dans des communes comme Pardailhan et Graissessac ${ }^{92}$ où plus de 6 foyers fiscaux sur 10 ne sont pas assujettis à l'impôt direct, le revenu moyen déclaré de ces foyers est inférieur à $9000 €^{93}$. Dans les autres villages, cette situation concerne un peu plus d'un foyer sur deux. À cette date, selon l'Insee, en France une personne est considérée comme pauvre lorsque son niveau de vie est inférieur à $954 €$ par mois. Une partie des foyers fiscaux non imposables de ces communes se retrouve donc dans cette définition.

53 «En France métropolitaine, le niveau de vie médian de la population s'élève à 19740 euros annuels ${ }^{94}$.» Or aucune des communes n'atteint ce montant. Celle qui s'en rapproche le plus est Pierrerue (18 162 €). Ensuite viennent Margon (17 717 $€$ ), Montouliers (17 $213 €)$, Graissessac (16 167 $€$ ) et enfin Pardailhan (13 $347 €)$ avec un niveau inférieur au montant du SMIG ${ }^{95}$.

Pourtant l'étude des résultats électoraux des cinq villages montre que les facteurs d'accroissement du chômage et le maintien de la précarité sont insuffisants à eux seuls pour expliquer le succès des votes $\mathrm{FN}$. Si un certain marasme économique ${ }^{96}$ et la flambée du chômage qui atteint 25 \% en 2014 peuvent apparaître comme des facteurs explicatifs à la brutale montée des votes frontistes à Graissessac en 2017, ils sont largement inopérants à Pardailhan où ce sont des taux d'abstention élevés récurrents qui marquent l'état de précarité d'une majorité d'habitants. On constate également que 
les scores FN les plus élevés se retrouvent à Margon, dans la commune la moins atteinte par la précarité.

Cette brève étude, en dépit de l'étroitesse de sa base, tend à montrer que des liens de corrélation ne sont pas nécessairement des liens de causalité. Sans nier l'importance du chômage et de la précarité, ni celle des répercussions psychologiques qu'elles engendrent, les résultats électoraux montrent que ces facteurs, malgré leur importance, ne peuvent être qu'une partie de l'explication de la montée des votes FN.

\subsubsection{L'explication par la spécificité géographique du vote FN}

Malgré un aspect accrocheur, susceptible d'une bonne diffusion dans l'esprit public, c'est la pertinence de l'objet même de ce genre de débat qui pose question puisqu'il présuppose l'existence d'un électorat frontiste rural, fût-il du nord ou du sud. Or, si les habitants des villages étudiés qui apportent leurs voix au FN sont bien des électeurs ruraux $^{102}$, ainsi nommés par opposition à ceux qui vivent et votent en ville, il n'est pas vrai qu'ils forment un électorat homogène. Comme cela a déjà été indiqué supra, l'instabilité des votes, en fonction du type d'élections et des différentes conjonctures dans lesquelles se déroulent les consultations, montre qu'il s'agit très majoritairement pour chaque scrutin de l'addition ponctuelle de votes individuels. 


\subsubsection{Une autre piste : les conséquences des mutations du monde agricole et la généralisation du salariat}

61 Jusqu'à la seconde moitié du $\mathrm{xx}^{\mathrm{e}}$ siècle, à l'exception des villages miniers et de quelques villages manufacturiers, la population d'un village languedocien était composée en majorité de paysans, quelques-uns propriétaires, la majorité ouvriers agricoles possédant souvent en propre quelques arpents de terre. Il existait des structures fédératives puissantes comme les coopératives vinicoles, des sociabilités locales fortes, certaines politisées, d'autres seulement traditionnelles comme les fêtes votives qui réunissaient la communauté villageoise dans des activités communes ou festives, échelonnées au cours l'année. La couleur politique du maire et de l'équipe municipale, notamment dans les mairies communistes, renforçait une tendance au vote communautaire ${ }^{103}$. Ce n'est plus le cas actuellement. Dans les villages, les agriculteurs, comme le montrent les chiffres clés de l'Insee, sont désormais minoritaires ${ }^{104}$. Le village n'est plus le lieu d'une communauté paysanne. Le phénomène semble irréversible ${ }^{105}$.

L'agriculture, fruitière et pastorale dans les régions montagneuses ou vinicoles en plaine, est confrontée à de profonds bouleversements et connait une baisse drastique des exploitations. Le nombre des exploitants actifs a été divisé par trois depuis 1970, un processus qui s'est accéléré depuis l'an $2000^{106}$, ainsi que le vieillissement des chefs d'exploitation ${ }^{107}$. Un tel recul a des conséquences économiques et sociologiques. Un nombre toujours croissant d'habitants, y compris parmi les natifs, sont maintenant des employés dépendant du secteur tertiaire. L'étude des documents de l'Insee depuis 1999 montre une généralisation du processus plus ou moins accéléré selon les communes. Des cinq villages, c'est Margon qui connait la progression la plus spectaculaire. En 2013, le taux de salariat y atteint $77,7 \%$ pour les hommes et $85,3 \%$ pour les femmes ${ }^{108}$.

À ces mutations économiques s'ajoutent pour certains villages d'importants changements démographiques. L'ouest héraultais rural a connu des fluctuations de population importantes au cours $\mathrm{du} \mathrm{xx}^{\mathrm{e}}$ siècle. Il s'est longtemps caractérisé par un exode rural avant de voir le phénomène s'inverser après 1975. Si, jusqu'en 1999, la situation dans ces villages demeure très contrastée, par la suite la tendance à l'accroissement se généralise. Même incomplètement retransmis au niveau des inscriptions sur les listes électorales, cet accroissement se traduit par une augmentation de plus de $11 \%$ d'inscrits sur les listes de 2012 par rapport à $1981^{109}$.

Cet afflux de population modifie la composition des listes des électorales et perturbe les équilibres sociaux internes des villages. Il peut même être à l'origine de tensions liées à des phénomènes de périurbanisation caractérisées par la création de lotissements ${ }^{110}$. Les nouveaux venus, souvent ignorants du passé du village et peu intéressés par ses usages traditionnels, intègrent peu les anciennes sociabilités qui perdent de leur vigueur et disparaissent au profit d'autres structures de sociabilités qui ne remplissent plus le même rôle fédérateur de la communauté. Phénomène généralisé dans les campagnes, ce déclin des sociabilités traditionnelles, décrit en France du Nord par Emmanuel Pierru et Sébastien Vignon dans les villages de la Somme, " pousse les gens à investir essentiellement dans la sphère privée » et le vote rural perd son sens spécifique $^{111}$. Il n'est plus le fait d'une population agricole, il a aussi perdu ses réflexes communautaires. Il n'est plus représentatif du monde paysan, mais devient la somme des votes d'individus aux métiers et aux préoccupations diverses qui n'ont plus en commun que leur lieu de résidence et ses contraintes ${ }^{112}$. 
65 Le «désert rural» rend les habitants de plus en plus tributaires des villes ${ }^{113}$. La métropolisation des emplois et la fermeture des usines existantes dans ces cantons ruraux de l'Hérault accélèrent le passage au salariat tertiaire marchand, commerce et services, le plus souvent à des postes sans véritables qualifications spécifiques. Ce secteur est particulièrement sensible aux aléas de la conjoncture économique. Les CDD et le travail partiel constituent une part importante des emplois proposés, entraînant une insécurité psychologique et socio-économique devant une carrière qui s'annonce précaire. Cette insécurité de l'avenir, même sans précarité économique immédiate, engendre un besoin de sécurisation, exploitée électoralement par le FN de Marine Le Pen. On rejoint ici une analyse faite par Michel Bussi, Jérôme Fourquet et Céline Colange ${ }^{114}$ après les élections de 2012 à propos des petites villes situées à proximité de métropoles, là où se concentre une majorité de petits salariés ${ }^{115}$.

66 Au vu des résultats électoraux des cinq villages, mais aussi de l'ensemble des communes étudiées, une corrélation paraît donc exister entre un essor du salariat conjugué à la perte d'importance du secteur agricole et la montée des votes frontistes. C'est le cas à Margon, ainsi qu'à Graissessac, où le secteur tertiaire, qui a pris la relève d'un secteur minier et industriel en voie de disparition, est le principal pourvoyeur d'emplois ${ }^{116}$. Il en est de même pour les quatre communes en tête des scores en faveur de Marine Le Pen en avril $2012^{117}$. On constate que leurs taux de salariat avoisinent ou dépassent les $70 \%{ }^{118}$ à une exception près ${ }^{119}$.

67 Une étude des quelques communes ayant voté à moins de $10 \%$ en avril 2012 et en juin 2014 semble confirmer à rebours cette hypothèse. À Cassagnoles, le secteur agricole représente $80 \%$ en 2009 , et en 2013 encore $66,7 \%$ des entreprises actives du lieu. Les six autres villages concernés ${ }^{120}$, à une exception près ${ }^{121}$, ont un taux de salariat inférieur à $55 \%$ pour les hommes.

\section{Conclusion}

Il faut sans doute reprendre pour les électeurs ruraux la formule de Patrick Lehingue à propos du ralliement du monde ouvrier au FN : «Il serait honnête de dire qu'en la matière, ce qu'on sait c'est qu'on ne sait pas grand-chose » de ce que représente «la tentation du vote frontiste " pour un habitant de ces petites communes ${ }^{122}$. Sans doute les facteurs socio-économiques, ajoutés à d'autres comme «la désagrégation des écosystèmes communistes locaux » - la formule est de Julian Mischi123 - évoquée supra avec l'influence antérieure des mairies communistes sur le vote, jouent-ils leur rôle au moment du choix du bulletin à mettre dans l'urne. Il nous semble toutefois que la forte diminution des paysans (agriculteurs ou viticulteurs) dans les villages et le fait qu'une majorité d'habitants de ces communes exerce désormais des activités à tropisme urbain ne modifie pas seulement le bâti et le paysage, mais qu'il affecte aussi la qualification « rurale » du village et induit nécessairement un changement des mentalités.

69 Cette modification des états d'esprit conduit à des comportements électoraux différents. L'individualisation des votes affecte désormais tous les partis et tout particulièrement le $\mathrm{FN}$, qui ne peut se prévaloir d'aucun ancrage villageois antérieur. Le choix de l'électeur pour un candidat ou une liste FN se fait au moment de la consultation, moins en fonction de convictions idéologiques, qu'en fonction du ressenti de l'électeur sur un ou plusieurs aspects du programme du FN auquel il adhère et qu'il voudrait voir réaliser ${ }^{124}$. Cette conduite permet de comprendre la variété des raisons 
possibles et parfois contradictoires pour lesquelles un électeur peut voter pour un parti, au programme délibérément " attrape-tout », un parti qui présente l'avantage de n'avoir pas encore été confronté à l'exercice du pouvoir. Elle montre aussi la relative fragilité d'un véritable enracinement dans le temps.

Maintenu encore sur une trajectoire ascendante lors des élections législatives de juin 2017 avec, au second tour dans la $5^{\mathrm{e}}$ circonscription, comme en 2012, un candidat mariniste opposé au candidat LRM, il n'est toutefois pas certain que le FN est assuré de conserver à l'avenir ces afflux de voix qui convergent en sa faveur. Plusieurs failles internes au parti pourraient changer la donne et aboutir à détourner de la formation un certain nombre d'électeurs.

71 La première est l'absence constatée de structuration partisane locale. Le nombre des militants déclarés et des encartés, déjà très faible dans les cantons ruraux, n'augmente pratiquement pas. Dans ces petites communes, "où tout le monde se connaît", s'afficher frontiste, même mariniste malgré une dédiabolisation annoncée médiatiquement, oblige à assumer un choix que l'opinion villageoise continue de réprouver moralement ${ }^{125}$.

72 Une seconde faiblesse tient à la volatilité des candidatures frontistes. En dépit d'un effort de formation, le FN n'est pas encore parvenu à enrayer le phénomène de déperdition de ses candidats. Sur les 7 personnes qui se présentaient lors des cantonales en 2011, dont 5 étaient nouvellement encartées, aucune n'est candidate en mars 2015 lors des élections départementales ${ }^{126}$. Plus récemment, les derniers contacts locaux pris à l'occasion de l'élection présidentielle de 2017 montrent un désengagement ou un départ du parti de l'ensemble des candidats rencontrés lors des entretiens. Ce n'est peut-être pas obligatoirement une cause d'échec électoral, mais il s'agit incontestablement d'une fragilité structurelle.

73 Au-delà même de ces difficultés locales, c'est l'attractivité du parti frontiste qui pourrait s'avérer moindre après l'échec de Marine Le Pen à la présidentielle, précédé par une mauvaise prestation télévisuelle lors du débat d'avant second tour, auxquels se sont ajoutées les turbulences de l'été qui ont suivi avec le départ de Marion MaréchalLe Pen et la scission de Florian Philippot. Il n'est pas certain que la refondation et le changement de nom ${ }^{127}$, annoncé par Marine Le Pen lors du congrès de Lille le 10 mars 2018, peuvent redresser la situation. La place occupée par le FN, avec ou sans changement de nom, dans la recomposition toujours en cours du paysage politique français est largement inconnue. Au-delà des quelques élections partielles perdues, il sera sans doute possible d'en avoir un premier vrai aperçu en 2019, au moment des prochaines échéances électorales européennes.

\section{Annexes}


Répartition des 46 villages ciblés dans l'ouest héraultais. Le cartouche représente une vue de la ville de Béziers. 1 : Romiguières ; 2 : Lavalette ; 3 : Saint-Géniès-de-Varensal ; 4 : Graissessac ; 5 : Saint-Étienne-Estréchoux ; 6 : Le Pradal ; 7 : Carlencas-et-Levas ; 8 : Brénas ; 9 : Celle ; 10 :

Valmascle ; 11 : Villeneuvette ; 12 : Saint-Martin-de-l'Arçon ; 13 : Vieussan ; 14 : Cabrerolles ; 15 : Roquessels ; 16 : Montesquieu ; 17 : Vailhan ; 18 : Usclas d'Hérault ; 19 : Aumes ; 20 : Margon ; 21 Fouzilhon ; 22 : Caussignojouls ; 23 : Pailhès ; 24 : Causses-et-Veyran ; 25 : Roquebrun ; 26 :

Berlou ; 27 : Ferrières-Poussarou ; 28 : Saint-Vincent-d'Olargues ; 29 : Le Soulié ; 30 : Pardailhan ; 31 : Pierrerue ; 32 : Cazedarne ; 33 : Cébazan ; 34 : Poilhes ; 35 : Montels ; 36 : Montouliers ; 37 : Oupia ; 38 : Minerve ; 39 : La Livinière ; 40 : Félines-Minervois ; 41 : Vélieux ; 42 : Rieussec ; 43 : Verreries-de-Moussans ; 44 : Ferrals-lès-Montagnes ; 45 : Cassagnoles ; 46 : Boisset.

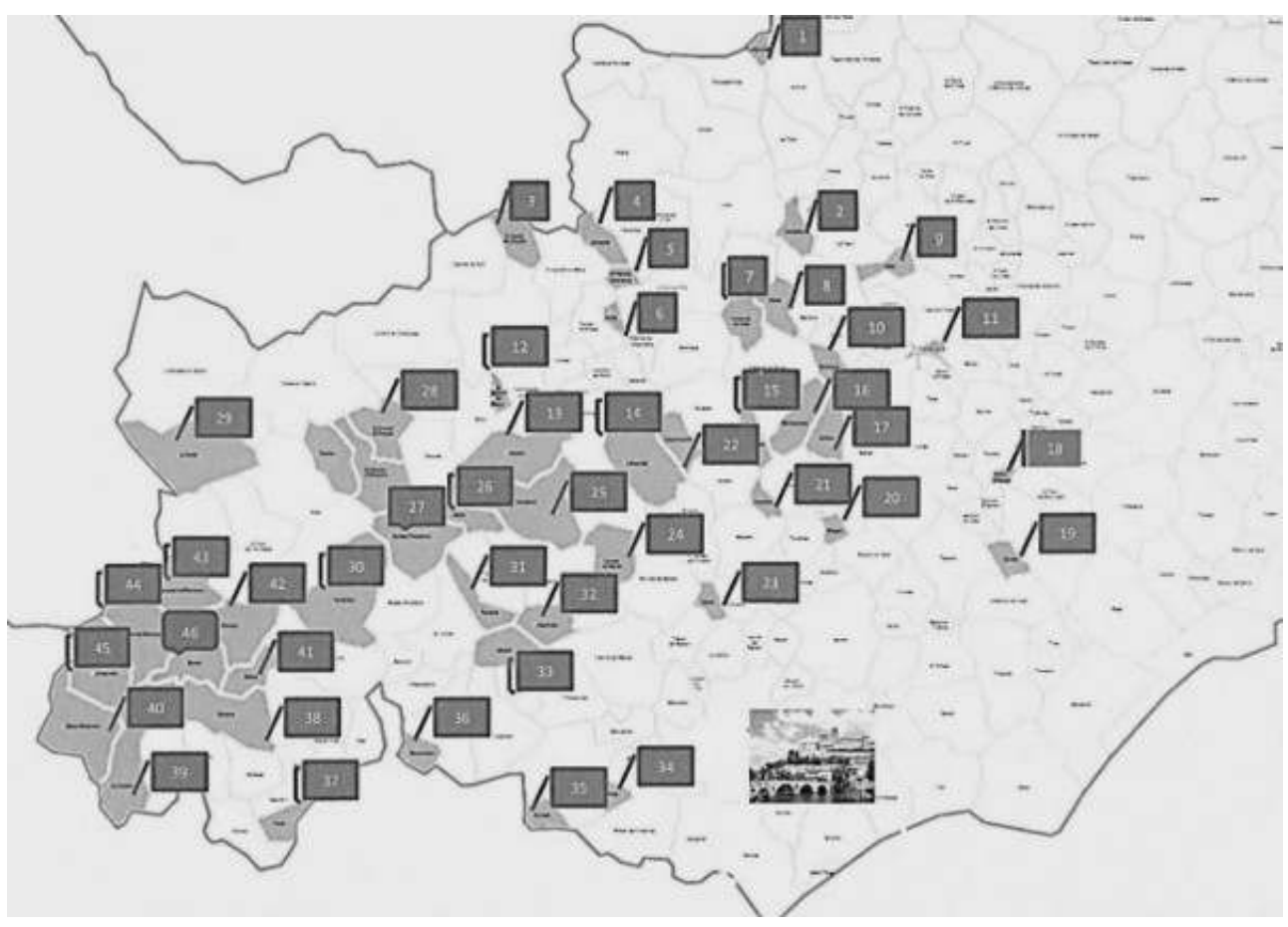

\begin{tabular}{|l|l|}
\hline \multicolumn{2}{|l|}{ Communes à scores FN élevés aux élections présidentielles (1988-2012) } \\
\hline 1988 & $\%$ FN \\
\hline Lavalette & 40,0 \\
\hline Ferrières P & 38,6 \\
\hline Romiguières & 35,7 \\
\hline Usclas-d'H. & 27,3 \\
\hline Fouzilhon & 26,2 \\
\hline Montels & 25,9 \\
\hline Caussiniojouls & 24,4 \\
\hline Valmascle & 24,3 \\
\hline Boisset & 22,6 \\
\hline
\end{tabular}




\begin{tabular}{|c|c|}
\hline Cabrerolles & 22,2 \\
\hline 1995 & $\% F N$ \\
\hline Ferrières $P$ & 28,6 \\
\hline Margon & 27,5 \\
\hline St-Étienne-E & 25,6 \\
\hline Fouzilhon & 25,4 \\
\hline Lavalette & 25 \\
\hline Brenas & 23,1 \\
\hline Montels & 22,1 \\
\hline Poilhes & 20,9 \\
\hline Usclas-d'H & 20,3 \\
\hline Carlencas-et-L & 19,6 \\
\hline 21 avril 2002 & $F N \%$ \\
\hline Margon & 35 \\
\hline Montels & 34,2 \\
\hline Usclas-d'H & 31,6 \\
\hline Boisset & 31 \\
\hline St-Étienne-E & 29,9 \\
\hline Fouzilhon & 28,9 \\
\hline Lavalette & 27,9 \\
\hline Romiguières & 27,3 \\
\hline Pailhès & 27,1 \\
\hline Valmascle & 26,5 \\
\hline 5 mai 2002 & $\% F N$ \\
\hline Romiguières & 52,94 \\
\hline Montels & 49,11 \\
\hline Lavalette & 41,46 \\
\hline
\end{tabular}




\begin{tabular}{|c|c|}
\hline Boisset & 35,71 \\
\hline Margon & 35,54 \\
\hline Vélieux & 35,29 \\
\hline Carlencas-et-L & 35 \\
\hline Usclas-d'H & 32,62 \\
\hline Poilhes & 32,35 \\
\hline St-Étienne-E & 30,46 \\
\hline 2007 & $\% F N$ \\
\hline Montels & 30,13 \\
\hline Fouzilhon & 27,45 \\
\hline Romiguières & 25 \\
\hline Montesquieu & 21,15 \\
\hline St-Étienne-E & 21,11 \\
\hline Boisset & 20,51 \\
\hline Valmascle & 20,41 \\
\hline Lavalette & 20,41 \\
\hline Pailhès & 20,22 \\
\hline Rieussec & 18,46 \\
\hline 2012 & $\% F N$ \\
\hline Valmascle & 36,95 \\
\hline Montels & 36,88 \\
\hline Fouzilhon & 33,75 \\
\hline Carlencas-et-L & 33,33 \\
\hline Romiguières & 33,33 \\
\hline Roquessels & 31,34 \\
\hline Poilhes & 30,34 \\
\hline Margon & 29,91 \\
\hline
\end{tabular}




\begin{tabular}{|l|l|}
\hline Lavalette & 29,55 \\
\hline Caussiniojouls & 28,89 \\
\hline
\end{tabular}

Évolution des pourcentages des voix FN dans les communes (élections présidentielles 1988 à 2017)

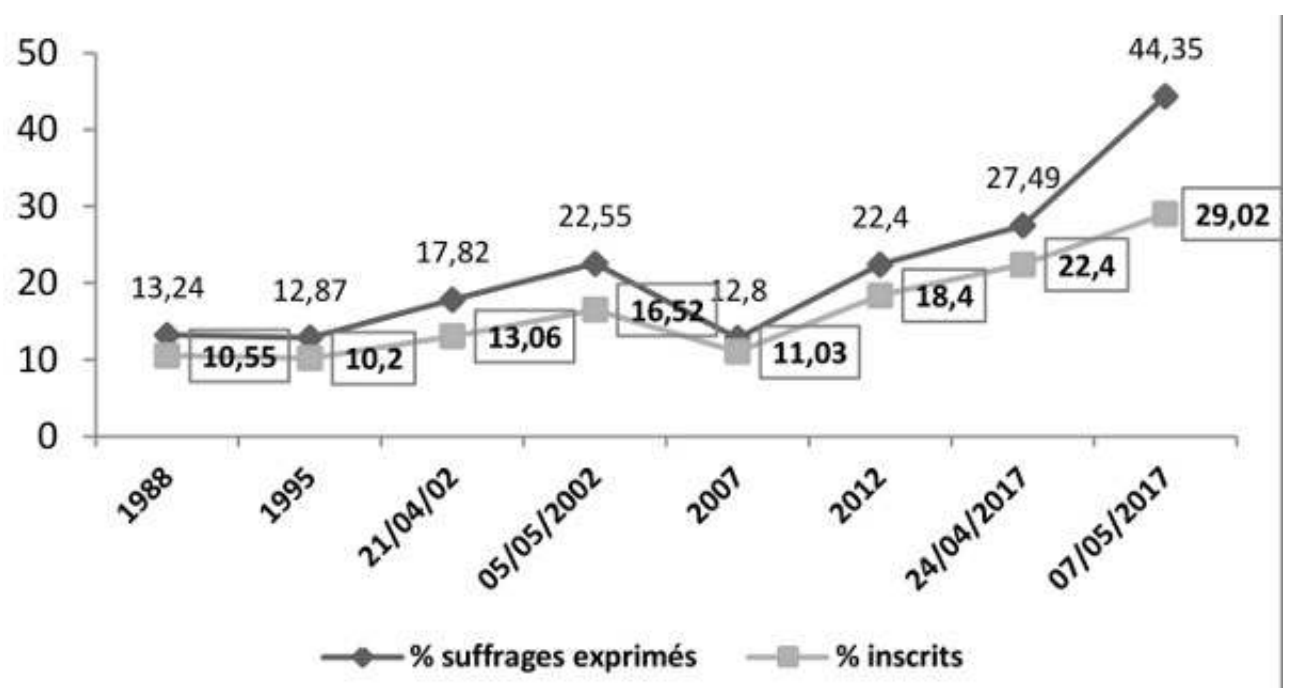

\section{NOTES}

2. L'étude comparée des résultats de Jean-Marie Le Pen et de Marine Le Pen lors des élections présidentielles à partir de 2002 a permis de définir la liste des communes sur un critère quantitatif. Ont été choisis les villages de moins de 500 électeurs les plus réceptifs $(+25 \%)$ et les plus réticents $(-15 \%)$ à un vote frontiste dans la circonscription choisie.

3. Marie-Paule Crochet-Théry, «1984-2014, Implantation du Front national dans les petites communes rurales de l'Ouest héraultais : émergence, consolidation, résistances. Étude électorale de quarante-six villages de moins de 500 électeurs en 2012 ", thèse de doctorat, université Paris Nanterre, 2017.

4. Bernard Schwengler, «Le vote d'extrême-droite en zone rurale : le cas de la France du Nord-Est », CEVIPOF, mars 2003, http://www.cevipof.com.

5. Emmanuel Pierru et Sébastien Vignon, «Déstabilisation des lieux d'intégration traditionnels et transformation de l'“entre-soi" rural. L'exemple du département de la Somme ", dans Céline Bessière, Éric Doidy, Olivier Jacquet, Gilles Laferté, Julia Mischi, Nicolas Rénathy, Yannick Sencébé (dir.), Les mondes ruraux à l'épreuve des sciences 
sociales, Versailles, Quae, 2007 ; Emmanuel Pierru et Sébastien Vignon, «L'inconnue de l'équation FN : ruralité et vote d'extrême droite. Quelques éléments à propos de la Somme ", dans Annie Antoine, Julian Mischi (dir.), Sociabilité et politique en milieu rural, Presses Universitaires de Rennes, 2008.

6. Elle venait juste d'avoir 20 ans.

7. «Cette expression est employée fréquemment par les historiens, les journalistes et les hommes politiques. L'expression fait surtout référence à un certain nombre d'événements ou de faits de mentalité s'étant produits dans la partie méridionale de la France [...] en particulier [dans les] quatre départements du Languedoc [et du] Roussillon. Seraient ainsi autant de manifestations du Midi rouge : la résistance au coup d'État de 1851, les communes de Marseille et de Narbonne, la révolte paysanne de 1907, les maquis de 1944 ou plus simplement les votes majoritaires de gauche depuis 1945 » (Jean Sagnes, Le « Midi rouge ». Mythe et réalité, Paris, Anthropos, 1982, p. 9.

8. Au soir du 26 avril 1981, dans l'ensemble des villages François Mitterrand obtient $30,05 \%$ des voix, Georges Marchais 24,08 \%. La gauche, hors scores des petits candidats, recueille pratiquement $55 \%$ des suffrages. Valéry Giscard-d'Estaing, le président sortant, n'en obtient que $20,17 \%$ et Jacques Chirac seulement 13,66 \%. La droite n'est majoritaire que dans 12 villages et Jacques Chirac ne l'emporte au second tour que dans 9 d'entre eux.

9. Lors de l'élection présidentielle de 1995, Robert Hue obtient 13,03\% des voix dans ces villages contre $8,3 \%$ au plan national et 10,24\% dans le département de l'Hérault.

10. Si Jean-Marie Le Pen y était arrivé en tête avec $17,83 \%$ des voix, son score restait inférieur de plus de 4 points à celui du département.

11. Jean-Marie Le Pen retrouva ses scores de 1995 et lors des législatives le FN recueillit seulement 5,79 \% des voix sur l'ensemble des communes ciblées.

12. Georges Frêche, exclu du PS, s'y présentait en vue de sa réélection à la présidence de région contre la candidate de son propre parti qu'il élimina dès le premier tour. Sa liste l'emporta largement au second tour avec cependant une abstention à 44,21 \% .

13. Fille d'Alain Jamet, l'un des fondateurs du FN avec Jean-Marie Le Pen en 1972.

14. $9,63 \%$.

15. "Comme je vous l'ai dit dans ces territoires il n'y a pas de gros problèmes d'insécurité, d'immigration massive ou d'islam radical.", entretien de Constance Calandri, candidate mariniste dans la $5^{\mathrm{e}}$ circonscription de l'Hérault pour les élections législatives de juin 2012.

16. Hormis les sénatoriales, où le faible nombre d'élus frontistes parmi les grands électeurs a rendu impossible jusqu'en 2014 toute représentation du parti dans cette assemblée, et les élections municipales à cause de la très grande difficulté d'interprétation des résultats au vu d'enjeux locaux pas toujours évidents à décrypter, auxquels s'ajoutent les spécificités du vote dans les communes de moins de mille habitants.

17. Le Midi Libre et le quotidien communiste La Marseillaise, édition de Béziers.

18. Emmanuel Négrier, « Le Pen et le peuple. Géopolitiques du vote FN en LanguedocRoussillon », Pôle Sud, vol. 37, n² 2, 2012, p. 153-166, https://www.cairn.info.

20. Le nombre de communes concernées. 
21. C'est-à-dire le rapport entre le nombre d'inscrits de ces communes et le total des inscrits de l'ensemble des villages pour mesurer leur "poids» dans l'évolution de l'ensemble des villages.

22. L'évolution des votes frontistes est directement liée aux différents types de scrutins, à leurs modes et à leurs enjeux, lesquels, outre la conjoncture socioéconomique du moment, varient en fonction de la nature, de la diversité et de l'étendue des pouvoirs de l'élu et de celle du territoire sur lequel ces pouvoirs s'exercent.

23. 5 élections durant la période considérée.

24. Voir annexes, tableau 1.

25. Ils représentaient 7,33 \% en 1988, 7,06\% en 1995, 15,03\% le 21 avril 2002 et $20,61 \%$ le 5 mai, $6,14 \%$ en $2007,18,85 \%$ en $2012,29,24 \%$ le 23 avril 2017 et $44,24 \%$ de l'ensemble des votes exprimés par les 46 communes.

26. Les départementales sont appelées cantonales avant le scrutin de 2015.

27. Hors les élections de 2007, particulièrement désastreuses pour le FN.

28. 7 élections eurent lieu entre 1986 et 2017. Voir les courbes supra.

29. Il gagne 6 communes entre 1989 et 2002.

30. Jusqu'en 1993, il s'agit de très petits villages. La majorité d'entre eux ne compte pas 100 inscrits et aucun ne dépasse les 200. Leur poids électoral est négligeable par rapport à l'ensemble des suffrages exprimés : 1,65 \% en 1988 et 2,19\% en 1993, 6,32 \% en 1997, $6,82 \%$ en 2002. Lors de l'effondrement des voix en 2007 une seule commune, Boisset, 45 inscrits, est au-dessus de $15 \%$.

31. $35,14 \%$ en suffrages exprimés, soit $20,37 \%$ des votes en se référant au nombre d'inscrits.

32. 41 sur 46 .

33. Caussignojouls ( $56,45 \%)$, Usclas-d'Hérault ( $53,52 \%)$, Carlencas-et-Levas $(51,85 \%)$ et Le Soulié $(50,68 \%)$.

34. Cassagnoles, 3 voix, $8,57 \%$ des suffrages, et Celles, où le FN n'a obtenu aucun vote en sa faveur.

35. Gilles Ardinat.

36. Jusqu'en 2012, la $5^{\mathrm{e}}$ circonscription de l'Hérault, fief socialiste depuis des décennies, voit au second tour après l'effondrement de la candidate du PS un candidat LRM opposé à un candidat $\mathrm{FN}$.

37. «On distingue des élections de premier ordre dont le but est la dévolution du pouvoir central et des élections de second ordre dites intermédiaires qui se déroulent entre les élections fondamentales et dont l'objectif est le choix des représentants et des gouvernants au niveau local, régional ou européen » (Pierre Bréchon, « La signification de l'abstention électorale ", séminaire doctoral, Université libre de Bruxelles, 29 avril 2010, p. 1.

38. Durant toute la période étudiée, d'André Saumade à André Vézinhet, ce sont des présidents socialistes qui sont en place dans l'Hérault.

39. Le poids du conseiller de Saint-Chinian, le socialiste Robert Tropéano, sur les maires de sa circonscription, poids politique parce qu'économique, est clairement évoqué à plusieurs reprises lors des entretiens à Pierrerue en octobre 2014. 
40. La loi du 17 mai 2013 impose désormais pour les élections départementales un scrutin paritaire, binominal.

41. $16,25 \%$ contre $17,83 \%$.

42. Un phénomène qui s'accroît avec le renforcement des pouvoirs des présidents par la réforme de 2003.

43. Toutes ont eu lieu au scrutin majoritaire à 1 tour jusqu'en 1998 et à 2 tours depuis 2004.

44. Jacques Blanc fut élu président de la région Languedoc en partie grâce aux voix des conseillers FN. En retour, Alain Jamet devient vice-président de région de 1986 à 1992.

45. Lors du scrutin de 1992, 16 communes sont à plus de $15 \%$, dont 11 nouvelles et parmi celles-ci 6 conserveront des scores supérieurs à $15 \%$ lors des consultations ultérieures.

46. Soit $18,4 \%$ des voix par rapport aux inscrits, soit un pourcentage légèrement supérieur à celui atteint par Marine Le Pen lors des présidentielles de 2012.

47. Ces consultations, à propos d'une Europe bouc émissaire de bien des politiques en campagne lors des autres scrutins, sont marquées par un désintérêt récurrent des électeurs de tous bords dans les villages comme ailleurs.

48. Rapportés en nombre d'inscrits, pour éviter les aléas des abstentions, les pourcentages de vote des communes n'atteignent $5 \%$ qu'en 1986, s'effondrent à $2,34 \%$ en 1999 et à 3,31 \% en 2009.

49. Fouzilhon, Montels et Romiguières.

50. Par ordre décroissant: Caussiniojouls, Fouzilhon, Valmascle, Saint-ÉtienneEstréchoux, Le Pradal, Margon et Montesquieu.

51. Il n'a pas été le seul. Le succès durant une décennie du CPNT, immédiat lors de sa création en 1989, celui plus éphémère des partis souverainistes en 1999, montre l'existence d'un important hiatus entre les électeurs ruraux et les partis classiques de gouvernement.

52. Le phénomène est patent lors des élections législatives du 9 juin 2002, avec des scores en forte baisse par rapport à celles du 25 mai 1997. À Caussignojouls par exemple, les scores passent de $23,88 \%$ à $10,81 \%$ - on le constate également lors des régionales du 14 mars 2010 par rapport à celles du 28 mars 2004. À Margon, en 5 ans, le FN perd près de la moitié de ses électeurs. En 2002, la perte des voix avait été à l'avantage de la droite. En 2010 elle se fait en faveur de la gauche (liste Georges Frêche).

53. Patrick Lehingue, «L'électorat du Front national. Retour sur deux ou trois idées reçues ", dans Gérard Mauger, Willy Pelletier (dir.), Les classes populaires et le FN. Explications de vote, Vulaines-sur-Seine, Le Croquant, 2016, p. 21.

54. Diminution par rapport aux tendances à la hausse observées dans les autres villages.

55. En 2014, lors des élections européennes et en 2015 lors du $2^{\mathrm{e}}$ tour des élections régionales, où l'on constate une baisse par rapport aux taux d'abstention du $1^{\mathrm{er}}$ tour.

56. Il s'agit de Margon, Montouliers, Pierrerue et Pardailhan.

57. Date de la $1^{\text {re }}$ élection régionale prise en compte.

58. À Pierrerue, l'avantage de Marine Le Pen, à $26,34 \%$, est de 6 points avec Macron dont le score est de $20,73 \%$. Il est de 8 points avec Fillon et Mélenchon, qui sont à 
égalité à $18,65 \%$. À Margon, l'écart est de pratiquement 20 points avec le second, Mélenchon (38,24 \% pour Marine Le Pen et 18,87 \% pour Mélenchon).

59. «Petit » en raison des faibles pourcentages de voix recueillies.

60. En 1999, une intervention du sénateur Gérard Delfau à l'adresse de la ministre de l'Aménagement du territoire et de l'Environnement, Dominique Voynet, n'hésite pas à employer le terme de "sous-développement» pour caractériser la situation de désindustrialisation de Graissessac qui suivit la fermeture des mines (Reconversion du bassin minier de Graissessac $11^{\mathrm{e}}$ législature - Question orale sans débat $\mathrm{n}^{\circ} 0465 \mathrm{~S}$ de Gérard Delfau).

61. $12,97 \%$ en 2007 et $19,95 \%$ en $2012,24,73 \%$ le 23 avril 2017 et $43,59 \%$ le 7 mai (abstentions : 28,18\%, blancs : 15,99\%).

62. Hausse qui reprendra avec vigueur en 2015 (30,74 \%).

63. Selon l'Insee, la variation annuelle moyenne est de $+6,8 \%$ entre 1999 et 2012 et atteint $+13 \%$ entre 2007 et 2013 .

64. Le score en 2012 est inférieur toutefois à celui de 2002, qui était de 36,67 \% le 5 mai.

65. 21,64 \% le 23 avril 2017.

66. Taux d'abstention : $27,12 \%$, bulletins blancs : $8,53 \%$.

67. C'est aussi un village où le CPNT recueille entre $15 \%$ et $20 \%$ en 1992, 1998 et 2004.

68. Résultats du 23 avril 2017 : Mélenchon, 29,1\%; Hamon, 3,73\%; Fillon, 8,96\%; Macron, 24,59 \% ; Le Pen, 21,64 \% (abstentions : 23,75 \%).

69. Le plus faible score est réalisé en 2002 avec $26 \%$ pour Lionel Jospin.

70. Insee, chiffres clés 2014.

71. Les partisans de la nature (scores des écologistes et du CPNT additionnés) avaient remporté presque $40 \%$ des suffrages. Dès 1986, les partis écologistes et pro-nature font à Pardailhan de bons scores. En 2010 encore, l'addition des écologistes indépendants et d'Europe-Écologie-Les Verts atteint 31,39\% des voix.

72. $28,26 \%$ au $2^{\text {e }}$ tour avec $19,4 \%$ d'abstentions et $12,04 \%$ de bulletins blancs.

73. Résultats du 23 avril 2007 : Mélenchon, 27,27\% ; Hamon, 10,91 \% ; Fillon, 9,09 \%; Macron, $24,55 \%$; Le Pen, $12,73 \%$.

74. Une inversion des votes qui conforte l'idée de l'impact à la fois économique et politique du Conseil régional et des hommes en place dans les choix des électeurs.

75. 280 contre 289 habitants.

76. Insee, chiffres clés.

77. 13,33 \% le 28 mars 2004, 16,24 \% le 14 mars 2010, 20,28 \% le 21 mars 2010.

78. En 1992, Pierrerue, avec $21,95 \%$ pour le CPNT, signe le score le plus important des 5 villages.

79. $51,15 \%$ au $1^{\text {er }}$ tour, $60,9 \%$ au $2^{\mathrm{e}}$ tour lors des élections de 2010 .

80. Alexandre Dézé « La construction médiatique de la "nouveauté" FN », dans Sylvain Crépon, Alexandre Dézé, Nonna Mayer (dir.), Lesfaux semblants du Front national. Sociologie d'un parti politique, Paris, Presses de Science Po, 2015, p. 455-504.

81. Par opposition à la France citadine et aux proches banlieues.

82. Pascal Perrineau, La France au Front, Paris, Fayard, 2014, p. 113.

83. Christophe Guilluy, Fractures françaises, Paris, François Bourin, 2010, p. 113-118. 
84. «Les chiffres-clés de la DRJSCS, Languedoc-Roussillon », mars 2014, http:// occitanie.drjscs.gouv.fr. Le taux de pauvreté des jeunes de 20 à 24 ans atteint 24,6\%.

85. « Note d'analyse : la pauvreté en milieu rural », https://www.cnle.gouv.fr.

86. En 2012, la liste des villes « pôles de services » où les habitants des villages peuvent avoir accès aux équipements et services les plus courants montre une nette disparité d'accès aux services, au profit de l'est de la zone étudiée qui a bénéficié de la construction de l'autoroute A 75, au détriment des cantons de montagne et de celui d'olonzac plus isolés.

87. Taux de pauvreté des petites villes, pôles économiques dans la zone étudiée : Lodève, $26 \%$; Pézenas, $26 \%$; Clermont-l'Hérault 21,4 \% ; Bédarieux, 18,9\%.

88. $15,2 \%$ au $2^{\mathrm{e}}$ trimestre 2013.

89. Le terme "foyer fiscal » désigne l'ensemble des personnes inscrites sur une même déclaration de revenus. Il peut y avoir plusieurs foyers fiscaux dans un seul ménage (définition Insee).

90. Pour la $1^{\text {re }}$ fois, à cette date, plus de la moitié des foyers fiscaux de Margon sont imposables $(52,4 \%)$.

91. Montouliers, 42,6 \% ; Graissessac, 45,4 \% ; Pierrerue, 47,6\%.

92. Graissessac, 63,5\% ; Pardailhan, 65 \% (source : Insee).

93. $8148 €$ à Pardailhan, $8976 €$ à Graissessac.

94. « Les niveaux de vie en 2012 », Insee, http://www.Insee.fr.

95. Montant du SMIG brut mensuel en 2012 : $1398,37 €$ (source : Insee).

96. À Graissessac, le nombre de créations d'entreprises, après une brève embellie en 2014, s'est complètement effondré alors que monte en 2017 le nombre des entreprises radiées. Les agences immobilières, les organisations associatives et sportives représentent près de $80 \%$ des secteurs d'activité les mieux représentés en 2018. « Entreprises à Graissessac (34260) », Le Figaro, http://entreprises.lefigaro.fr.

97. Hervé Le Bras, Une autre France, votes, réseaux de communication et classes sociales, Paris, Odile Jacob, 2013. La démonstration appuyée sur des similitudes cartographiques donne lieu à l'établissement d'un gradient mesurable de diffusion des idées lepénistes depuis les centres-villes jusqu'à la périphérie rurale. Cette hypothèse, objet de controverses, ne correspond de toute façon plus à la situation actuelle de l'implantation du FN.

98. Tous les entretiens, réalisés tant à Margon qu'à Pierrerue, montrent combien le parcours de vie individuel reste déterminant et que, dans la grande majorité des cas, c'est un élément factuel survenu dans leur vie personnelle qui entraine en réaction le passage à l'acte de voter et de militer.

99. Il est par exemple évident que la mobilité des habitants des villages est souvent contrainte par l'obligation d'aller travailler là où se trouve les emplois et que le lieu du logement obéit très souvent à des logiques économiques plutôt qu'à des choix de vie.

100. Octave Nitkowski, Le Front national des villes et le Front national des champs, Paris, Jacob-Duvernet, 2013. Son blog est hébergé par le quotidien Libération.

101. Pour la réfutation de cette théorie, voir Joël Combin, «Les trois visages du vote FN », Le Monde diplomatique, décembre 2015, p. 1 et 6. 
102. Un second questionnement pourrait avoir lieu sur le sens à donner au mot rural, tant les mutations économiques et démographiques qui ont affecté ces villages ont été profondes.

103. On n'était pas obligatoirement communiste, mais on votait communiste pour la défense de la vigne et de la petite propriété.

104. Dans ces conditions, ce n'est sans doute pas un hasard si, lors des entretiens, les seuls agriculteurs rencontrés étaient des retraités. Leurs enfants étaient des employés.

105. Bertrand Hervieu et François Purseigle, Sociologie des mondes agricoles, Paris, Armand Colin, 2013. Ils insistent sur l'urgence et la nécessité d'une nouvelle approche sociologique du monde agricole devant l'éventualité de la disparition de l'objet même de recherche.

106. La vigne recule sous l'action conjuguée des arrachages coordonnés par Bruxelles et des modifications climatiques. Quant aux vergers et aux productions arboricoles, selon les données du ministère de l'Agriculture en 2012, elles-mêmes issues du recensement agricole de 2010 (www.agreste.agriculture.gouv.fr), leur surface est également en baisse et le secteur connaît des crises successives liées à la concurrence internationale, notamment espagnole.

107. Évolution du nombre de chefs d'exploitation actifs dans le département de l'Hérault : en 1970, 34703 ; en 2000, 16251 ; en 2010, 10 815, dont 1740 âgés de moins de 40 ans et 3502 âgés de plus de 60 ans (Source: Agreste, Recensements agricoles 1970, $1979,1988,2000,2010)$.

108. Insee, chiffres clés, 23 octobre 2014.

109. Chiffre obtenu en comparant le nombre officiel des inscrits lors du premier tour des élections présidentielles à ces 2 dates. Voir http://www.interieur.gouv.fr.

110. Ainsi, la commune de Margon a vu sa population passer de 209 habitants en 1999 à 571 en 2011. Lors de l'entretien du 25 septembre 2014, les participants déplorent que « la construction des maisons se soit faite sur des parcelles trop exiguës » : « Source de meilleure rentabilité pour les promoteurs, ce découpage a eu pour effet de scinder les gens du village en deux catégories, "les riches" qui habitent au cœur de la commune, près du château, et "les autres" qui habitent les lotissements ». À noter que parmi les 3 locuteurs, le premier habite un lotissement, 1 autre près du château, le $3^{e}$ dans un village voisin et que tous partagent la même analyse.

111. Emmanuel Pierru et Sébastien Vignon, «Comprendre les votes frontistes dans les mondes ruraux ", dans Les classes populaires et le FN, op. cit., p. 89.

112. Anciens habitants de la commune ou nouveaux venus, après la fermeture progressive des petits commerces, les villageois assistent impuissants depuis plusieurs années à une déstructuration programmée de leur cadre de vie, avec la disparition des bureaux de poste, de classes dans le primaire et de cliniques, ainsi que des médecins généralistes.

113. Margon est désormais classé par l'Insee en « aire urbaine multipolaire ». En 2009, le nombre des actifs travaillant hors de la commune s'élevait à $80,11 \%$. Le secteur agricole pour cette commune viticole ne représente plus que $16,1 \%$ des établissements actifs en 2013 (Insee, chiffre clés, 2014). 
114. "Analyse et compréhension du vote lors des élections présidentielles de 2012. L'apport de la géographie électorale ", Revue française de science politique, vol. 62, $\mathrm{n}^{\circ}$ 5-6, 2012, p. 941-964.

115. Entre $20 \mathrm{~km}$ et $50 \mathrm{~km}$.

116. En 2012, il est de $74,8 \%$ pour les hommes et $85,7 \%$ pour les femmes (Insee, chiffres clés, 2014).

117. Carlencas-et-Levas, Montels, Fouzilhon et Valmascle.

118. De 69,5\% à Montels à 76,9 \% à Carlencas-et-Levas (Insee, op. cit.).

119. Fouzillon à $54,6 \%$ (ibid.).

120. Brenas, Celles, Minerve, Vieussan, Saint-Martin-de-l'Arçon et Villeneuvette.

121. Saint-Martin-de-L'Arçon.

122. Patrick Lehingue, art. cité, p. 31-33.

123. Julian Mischi, «Essor du FN et décomposition de la gauche en milieu populaire ", dans Les classes populaires et le FN, op. cit., p. 123.

124. Tous les entretiens confortent ce point de vue. Chaque interlocuteur explique son choix de voter Marine Le Pen et les candidats qui s'en réclament mettent en avant un ou plusieurs aspects du programme. Il arrive alors que des points de vue contradictoires se manifestent autour d'une même table, les entretiens ayant été menés au cours de réunions de 2 à 5 personnes, afin de créer une interactivité.

125. « Je vais être honnête avec toi, je ne vais pas être sur la liste avec toi, car je ne veux pas passer pour un extrême", dit en 2014 un ami du candidat RBM à la mairie de Pierrerue.

126. Une recherche systématique dans les autres cantons montre qu'aucun d'entre eux ne s'est présenté ailleurs.

127. Le FN deviendrait le Rassemblement national (RN) après ratification d'un vote des militants par courrier.

\section{RÉSUMÉS}

L'étude des votes frontistes entre 1984 et 2017 dans quarante-six petites communes rurales de l'Ouest héraultais, complétée par un travail monographique sur cinq d'entre-elles, montre un succès limité du FN jusqu'en 2011, suivi d'une progression linéaire jusque 2017. Sans sous-estimer le rôle joué par les difficultés socio-économiques du monde périphérique rural, les mutations sociologiques de la population de ces villages, composée de plus en plus majoritairement par des salariés du secteur tertiaire, apparaissent comme l'un des facteurs explicatifs de la croissance des votes en faveur du FN.

Study of the votes supporting the National Front party between 1984 and 2017 in forty six small rural districts on the west of the department of the Hérault, completed by a monographic work on five of them, schows a success limited for FN until 2011, followed by a linear progress to 2017. Without underestimating the importance of the role played in this development by the socio- 
economic difficulties of the rural peripheral world, the deep sociological changes of these communities, more and more mainly made up of employees of the tertiary sector, appear as one of factors explaining of the growth of the FN votes.

INDEX

Mots-clés : commune, front national, rural, mutation, Hérault

Keywords : district, national front, rural, change, Hérault

\section{AUTEUR}

\section{MARIE-PAULE CROCHET-THÉRY}

Docteure en science politique, université Paris Nanterre École doctorale de droit et science politique (DSP) 